\title{
Diagnostic and prognostic serum marker of cholangiocarcinoma (Review)
}

\author{
XIAOJUN ZENG $^{1}$ and HUALIN TAO ${ }^{2}$ \\ ${ }^{1}$ Department of Laboratory Medicine, Luzhou Medical College; ${ }^{2}$ Department of Laboratory Medicine, \\ Affiliated Hospital of Luzhou Medical College, Luzhou, Sichuan 646000, P.R. China
}

Received February 9, 2014; Accepted October 24, 2014

DOI: $10.3892 / \mathrm{ol} .2014 .2696$

\begin{abstract}
Cholangiocarcinoma (CCA) is a fatal disease that is typically diagnosed late and treated ineffectively. As the morbidity and mortality rates for CCA rise markedly, patietns with CCA currently have a poor prognosis. However, if it were possible to diagnose CCA early while effective treat methods are available, CCA patients would achieve a better quality of life. Therefore, preventing the process of CCA in the early stages is an urgent problem to solve. An accurate, quick and safe method to diagnose early-stage CCA is required. The present review discusses the risk factors, status of research and certain serum markers of CCA. The sensitivity and specificity of these markers differ from each other. To explore the more accurate serum markers may be a novel direction and method for the diagnosis of CCA in laboratory medicine in the future.
\end{abstract}

\section{Contents}

1. Introduction

2. Risk factors

3. Status of research

4. Serum makers

5. Summary

\section{Introduction}

At present, the term cholangiocarcinoma (CCA) includes all bile duct cancers, consisting of $20 \%$ intrahepatic, $~ 55 \%$ perihilar, a subset of extrahepatic CCAs which involves the bifurcation of the ducts, $20 \%$ distal extrahepatic CCA, and

Correspondence to: Professor Hualin Tao, Department of Laboratory Medicine, Affiliated Hospital of Luzhou Medical College, 25 Taiping Street, Luzhou, Sichuan 646000, P.R. China E-mail: 1zyxyjyx@163.com

Key words: cholangiocarcinoma, serum marker, diagnostic, prognostic the remaining 5\% of CCAs are multifocal (1). CCA is an aggressive malignancy that, due to early invasion, widespread metastasis and the lack of an effective therapy, is associated with a high incidence and mortality rate (2). While CCA is relatively rare, the incidence rates have increased markedly worldwide in the past decade (3). In Thailand, particularly in the Northeastern region, CCA is a crucial health problem that is caused by a combination of Opisthorchis viverrini (OV) infection and nitrosamine (4). This trend in the incidence of CCA is followed by other regions of Southeast Asia and China (5). In England and Wales, from 1997-1998, CCA caused almost 1,000 mortalities per year (6).

\section{Risk factors}

In intrahepatic cholangiocarcinoma (ICC), several risk factors have been established, including primary sclerosing cholangitis (PSC), fibropolycystic liver disease, parasitic infection, intrahepatic biliary stones and chemical carcinogen exposure. Tanaka et al (7) performed a large-scale cohort study in the province of Osaka (Japan) and found that hepatitis B virus (HBV) infection and liver inflammation are independently associated with ICC development, even though there remains a requirement for further large cohort studies to verify these findings. A meta-analysis by Li et al revealed that $\mathrm{HBV}$ is associated with an increased risk of CCA, particularly for ICC (8). As the incidence of ICC is rising with the emergence of hepatitis $\mathrm{C}$ virus (HCV), Sempoux et al (9) considered that HCV infection is also a risk factor.

In perihilar cholangiocarcinoma, a variety of risk factors have been identified, including advanced age, male gender, PSC, choledochal cysts, cholelithiasis, cholecystitis, parasitic infection, including with OV and Clonorchis sinensis, inflammatory bowel disease, alcoholic and nonalcoholic cirrhosis, chronic pancreatitis and metabolic syndrome (10).

For extrahepatic cholangiocarcinoma (ECC), studies reporting the risk factors appear to be fewer compared with those for ICC and perihilar CCA. However, it is indicated that blood type $\mathrm{A}$ is more common in younger ECC patients, and there is a lower proportion of serum carbohydrate antigen (CA)-125 and elevation of CA19-9 in patients with blood type A. Blood type A and HBV infection are associated with ECC risk, and there is a synergism of blood type A and HBV infection in the development of ECC (11). 


\section{Status of research}

As the incidence of CCA is rapidly increasing, numerous investigations focus on this disease. According to experimental evidence, CCA can be classified into three types, CCA cell lines, animal models, and human tissue, blood and bodily fluid. Currently, a large amount of CCA data, covering all fields of this disease, has been published and involves studies into genes (12-14), proteins (14), metabolic enzymes (15), signaling pathways (13) and specific cells (16-19).

\section{Serum makers}

CCA is associated with a late presentation and difficult diagnosis (20). At present, a confirmed diagnosis of CCA continues to rely on histopathological examination, an invasive method, despite the sample being difficult to obtain. The sample for another important examination, cytology, is also difficult to obtain and these methods possess the same risk of seeding (21). This emphasizes the need for serum tumor markers that are safe and exhibit high specificity and sensitivity.

CA19-9. CA19-9 is a serum tumor marker for pancreatic cancer, CCA and other malignancies with a suboptimal sensitivity and specificity. Li et al (22) found that the sensitivity and specificity of CA19-9 are 68.4 and $75 \%$, respectively. Leelawat $e$ t al considered the sensitivity and specificity of serum CA19-9 as a serum marker, with a cut-off value of 100 units $/ \mathrm{ml}$, to be 68 and $87 \%$, respectively. In conclusion, the accuracy of CA19-9 in identifying CCA is not high (23).

Singh et al (24) also found that in CCA patients, CA19-9 exhibited poor clinical utility as a tumor marker and did not alter patient management. The elevation in CA19-9 was suggested by the results to be associated with biliary obstruction based on clinical history, laboratory data and diagnoses. In addition, the elevated levels of serum CA19-9 and incomplete removal of stones were potential predictive factors for CCA in patients with hepatolithiasis (25). These results may indicate that CA19-9 is associated with biliary obstruction, but a large-scale clinic investigation is required to verify the findings.

Although CA19-9 serves as a serum maker for CCA, it is not satisfactory and there may be certain factors affecting the value. For example, allelic variants of fucosyltransferase (FUT) 2 and 3 affect the serum levels of CA19-9. In PSC patients, FUT2 and 3 levels are a screening parameter commonly used to evaluate the biliary malignancy. Based on the different genotypes of FUT3 and FUT2, which can determine the cut-off level of CA19-9, and the level of CA19-9 in 433 PSC patients, 41 of whom possessed biliary malignancy, Wannhoff et al (26) classified the PSC patients into three groups (no FUT3 activity regardless of FUT2 activity, both FUT2 and FUT3 activity, and no FUT2 activity without loss of FUT3 activity, respectively). Youden's index and receiver operating characteristic curve (ROC curve) revealed that the exclusive cut-off values for each group could increase the sensitivity to $90 \%$ and reduce the false positive results. Previously, Sinakos et al (27) also found that numerous patients with PSC and increased serum levels of CA19-9 did not suffer from CCA. In conclusion, when determining the level of CA19-9 in
PSC patients, the cut-off value should be modulated based on FUT2/3. Therefore, additional studies are required to explore whether there are other factors affecting CA19-9 levels.

CA19-9 is not only used for diagnosis, but also for prediction. A meta-analysis was performed to determine the prognostic role of pre-operative serum CA19-9 levels in the survival of patients with CCA. The results revealed that a pre-operative elevation in the CA19-9 levels of CCA patients was correlated with a poor prognosis (28).

In hilar CCA, a subgroup of CCA, the combination of CA19-9 and carcinoembryonic antigen (CEA) serum levels are associated with tumor stage. The high pre-operative serum levels of CA19-9 and CEA in hilar CCA patients demonstrated a decreased survival time and an increased incidence of irresectability (29).

S121 or CCA-CA and CA-S27. Silsirivanit et al (30) established a novel monoclonal antibody (MoAb) using pooled CCA tissue, they obtained an S121 immunoglobulin M MoAb that recognized a novel glycan epitope. Their findings demonstrated that mucin 5AC (MUC5AC) is a core glycoprotein for the S121 epitope. The serum S121 level was able to differentiate CCA patients from healthy individuals, active OV-infected individuals and patients with various gastrointestinal cancers, hepatoma and benign hepatobiliary diseases with high sensitivity $(87.63 \%)$, specificity $(89.58 \%)$, positive predictive value $(80.95 \%)$ and negative predictive value (93.47\%). High serum S121 levels were also associated with a poor patient outcome. In addition, the results from a golden hamster model of CCA suggested that CCA-CA is a potential marker for the early diagnosis of CCA (31).

CA-S27, is a novel Lewis a (Lea)-associated carbohydrate epitope and modification of MUC5AC mucin. A high level of CA-S27 was detected in the serum of CCA patients and was able to differentiate CCA patients from patients with gastrointestinal cancers, hepatomas, benign hepatobiliary diseases and healthy subjects, exhibiting a high sensitivity $(87.5 \%)$ and high negative predictive value (90.4\%). Also, patients with high serum CA-S27 exhibited a significantly shorter survival time compared with those possessing a low serum CA-S27 level, regardless of the serum MUC5AC levels. Notably, FUT3 was also revealed to be a regulator of CA-S27 expression. CA-S27 is a potential diagnostic and prognostic marker in CCA (32).

CA242.CA242 is a sialiated carbohydrate antigen that has been used as a tumor marker in several tumors. The combination of $\alpha$-fetoprotein(-) and CA242(+) afforded a high specificity (94.3\%) and a high accuracy (78.5\%) when distinguishing ICC from HCC (hepatocellular carcinoma) (33).

Matrix metalloproteinase (MMP)-7. Serum levels of MMP-7 were significantly elevated in CCA patients $(\mathrm{P}<0.001)$, which indicates that MMP-7 is a useful biomarker in differentiating CCA from benign biliary tract obstructive diseases (23). The sensitivity and specificity of serum MMP-7 is 53-76.32\% and $46.88-92 \%$, respectively, but at different cut-off values, this varies (Table I). ROC curve analysis revealed that the detection of the serum MMP-7 level is a reasonably accurate method for the differentiation of CCA from benign biliary tract disease patients [area under curve (AUC), 0.73-0.84] $(23,34,35)$. 
Table I. Sensitivity and specificity of serum MMP-7 at different cut-off values in distinguishing CCA from benign biliary tract disease.

\begin{tabular}{lcc}
$\begin{array}{l}\text { Cut-off value, } \\
\mathrm{ng} / \mathrm{ml}\end{array}$ & Sensitivity, $\%$ & Specificity, \% \\
\hline $5.5^{\mathrm{a}}$ & 75 & 78 \\
$6.0^{\mathrm{b}}$ & 76.32 & 46.88 \\
$6.5^{\mathrm{a}}$ & 63 & 87 \\
$7.4^{\mathrm{b}}$ & 63.16 & 71.88 \\
$7.5^{\mathrm{a}}$ & 53 & 92 \\
$17.0^{\mathrm{c}}$ & 75.8 & 72.5 \\
$23.0^{\mathrm{c}}$ & 55.9 & 83.5 \\
\hline
\end{tabular}

a59 CCA patients and 128 benign biliary tract diseases patients (23); ${ }^{\mathrm{b}} 44$ CCA patients and 36 benign biliary tract diseases patients (34); '37 CCA patients and 31 benign biliary disease patients (35). CCA, cholangiocarcinoma; MMP-7, matrix metalloproteinase-7.

The ROC curve analysis of another study revealed that the MMP-7 level could distinguish between CCA and other hepatobiliary diseases, including opisthorchiasis, benign biliary disease, HCC, hemangioma ( $\mathrm{P}<0.001$; AUC, 0.775) (35). However, in order to confirm whether MMP-7 could distinguish CCA from other cancers or biliary tract diseases, a large-cohort study would be required. Additionally, the process of setting up an accurate cut-off value for MMP-7 is also an issue.

Apolipoprotein (Apo)A-I and neutrophil gelatinase-associated lipocalin (NGAL). ApoA-I is a member of apolipoprotein A1/A4/E family that is synthesized in the liver and small intestine (36). Downregulated ApoA-I in CCA patients may be linked to liver and small intestine dysfunction. ApoA-I may differentiate CCA from benign diseases of hepatobiliary and healthy individuals (AUC, 0.883) and other cancers including laryngopharyngeal, laryngeal, esophageal, prostate and lung cancer (AUC, 0.715). Therefore, it is suggested that ApoA-I could be a potential useful biomarker for CCA (37).

NGAL is a $25-\mathrm{kDa}$ glycoprotein that belongs to the lipocalin superfamily (38) and regulates the action of gelatinase (39). Srisomsap et al (40) demonstrated that NGAL is a secreted protein that is only expressed in the conditioned media of the CCA cell line. Investigating NGAL expression in various types of cancer and normal tissues obtained from CCA patients revealed that NGAL was expressed only in the cancer tissues. High NGAL expression has been reported in various cancers and, among these cancers, the NGAL level was significantly elevated in CCA patients $(\mathrm{P}<0.001)$. The AUC of the ROC curve was 0.79 . NGAL is promising to serve as a biomarker to discriminate CCA patients from benign biliary tract disease patients (38).

al-antitrypsin (A1AT) and oxidized (ox)-A1AT. A1AT is a 52-kDa glycoprotein produced by hepatocytes and mononuclear phagocytes, and is the principal human inhibitor of neutrophil elastase (NE) (41). A1AT was found to be expressed in the tumors of CCA patients (42). In CCA patients, the plasma level of A1AT is upregulated and is significantly higher compared with control subjects. Serum levels of CA19-9 combined with $\mathrm{A} 1 \mathrm{AT}$ were the minimum requirement to obtain a predictive accuracy of $>80 \%$ in diagnosing CCA (43).

Ox-A1AT is a modified form of A1AT, which is generated by free radicals, released from inflammatory cells, oxidising the active site of A1AT. In patients with heavy OV infection, advanced periductal fibrosis (APF) and CCA, the serum levels of ox-A1AT were significantly higher compared with healthy controls $(\mathrm{P}<0.001)$. Ox-A1AT can therefore serve as a potential risk indicator for Opisthorchiasis-associated CCA (42).

KL-6 mucin. KL-6 mucin is a type of MUC1 mucin that may play an important role in unfavorable tumor behaviors, including the invasion and metastasis of carcinoma of the ampulla of Vater (44). In liver cancer tissues, KL-6 mucin is only expressed in ICC (45).

The serum KL-6 mucin level was elevated in ICC patients. When the cut-off value is set at 248 units $/ \mathrm{ml}$, the positive rate of ICC patients, healthy individuals and HCC patients is 100.0, 15.8 and $18.5 \%$, respectively. KL-6 is a potential serum maker for differentiating ICC from HCC (45).

Exostosin (EXT) 1. EXT1 is a novel biomarker that has been studied in CCA. Khoontawad et al (46) screened markers in the plasma of hamsters that were infected with OV and treated with N-nitrosodimethylamine (NDMA) to induce CCA. The most frequently upregulated protein, EXT1, was validated by western blot analysis. In human CCA, the plasma EXT1 level was significantly higher compared with healthy controls. The results of this study indicated that increased expression of EXT1 in the plasma may be involved in CCA genesis and be a potential biomarker of CCA.

$\alpha 1 \beta$-Glycoprotein $(A 1 B G)$ /afamin (AFM) ratio. A1BG was identified and confirmed as an elevated protein in pancreatic juice and cancer tissues of pancreatic ductal adenocarcinoma. A1BG may be a cancer-associated gene and a novel tumor marker for cancer (47). AFM, a member of the albumin family, is a vitamin E-binding protein (48) that was reported as a novel protein marker for ovarian cancer (49) and may be a negative acute-phase protein, although the pathophysiology is unclear at present (50).

A1BG and AFM were detected consistently at different degrees in CCA serum compared with controls. Tolek et al (51) selected the two types of protein using proteomes. The serum A1BG/AFM ratio has a high specificity (87.5\%) and sensitivity $(84.4 \%)$ in diagnosing CCA, as the ratio is significantly higher in CCA compared with the control.

Orosomucoid (ORM) 2 and kinesin (Kif) 18A. ORM2 is a key acute-phase plasma protein in inflammation and is reported to be a novel member of cancer-associated glycoproteins (52). Kif18A is a microtubule depolymerase, a key regulator of chromosome congregation and a member of the kinesin superfamily of molecular motor proteins (53). In hamster CCA models induced by NDMA, OV infection and OV + NDMA $(\mathrm{ON})$, respectively, Rucksaken et al (54) found that the plasma level of ORM2 was overexpressed in the OV and ON groups and Kif18A was overexpressed in the ON group at the 
precancerous stage, which was verified by western blotting. In conclusion, these results suggested that Orm2 and KIF18A may serve as potential biomarkers for early diagnosis of CCA.

Adhesion molecule (ICAM)-1. ICAM-1 is a member of the immunoglobulin superfamily of adhesion molecules and was reported to be associated with melanoma (55). ICAM-1 is important in cell-cell and cell-basement membrane interactions (56) and, in normal vascular endothelial cells and mononuclear macrophages located in the small intestine and lymph, ICAM-1 is expressed at low levels (57).

In CCA, the serum ICAM-1 level is significantly higher compared with healthy controls and exhibits high sensitivity (77\%) and specificity (70\%) at a cut-off value of $167 \mathrm{ng} / \mathrm{ml}$. Positive correlations between ICAM-1 and alkaline phosphatase and CEA levels were also identified. However, there was no significant difference between the serum ICAM-1 levels in CCA and benign biliary diseases with mainly inflammatory features. As the serum level of ICAM-1 significantly decreased following tumor resection, it may serve as a prognostic maker in CCA (58).

Pyruvate kinase isoenzyme type M2 (M2-PK). M2-PK is a novel tumor metabolic marker (59) that plays a key role in tumor metabolism and energy production by channeling glucose carbons, either into synthetic processes or toward glycolytic energy production. Increased aerobic glycolysis is one of the most common metabolic phenomenons in tumor cells, which particularly express M2-PK (60). As a result, aerobic glycolysis causes the accumulation of glycolytic phosphometabolites, allowing cells to invade areas with low concentrations of oxygen and glucose (61).

In CCA patients, the level of serum M2-PK was markedly higher compared with controls $(\mathrm{P}<0.05)$ and exhibited a high sensitivity $(84.2 \%)$ and specificity $(90 \%)$. M2-PK is maybe a promising maker in diagnosing CCA (22).

Hydroxyproline (HYP) and collagen I. HYP is an unique amino acid for maintaining cell structure and function, and is a major component of collagen (62). Excessive acids, MMPs and cathepsin K, could degrade collagen I into HYP (63). Prakobwong et al (35) found that the plasma levels of HYP and collagen I were significantly higher in opisthorchiasis patients compared with healthy individuals. The highest plasma level of HYP in the hepatobiliary disease patients was observed in CCA patients $(\mathrm{P}<0.05)$. The HYP and collagen I levels significantly differed between Opisthorchiasis, benign biliary disease and CCA patients $(\mathrm{P}<0.001)$. Notably, only the plasma HYP level in benign biliary disease was predictive of CCA risk (OR, 3.69; $\mathrm{P}=0.020)(35)$.

Golgi membrane protein (GOLM) 1. GOLM1 is transmembrane protein that is expressed in the Golgi of epithelial cells. Previous studies have indicated that the expression of GOLM1 is upregulated in acute and chronic liver diseases (64) and is useful in the diagnosis and therapy of prostate cancer (65). The overexpression of GOLM1 is associated with poor prognosis in human HCC (66). Kristiansen et al (64) used a membrane protein enrichment strategy in addition to ${ }^{18} \mathrm{O}$ labeling-based quantitative proteomics to identify proteins that are highly expressed in CCA. This study confirmed GOLM1, Annexin IV and epidermal growth factor receptor pathway substrate (Eps8) to be candidate biomarkers for CCA. Among these candidates, GOLM1 is detectable in serum and it is potentially able to serve as a biomarker for the early detection of CCA.

Angiotensin-converting enzyme (ACE). ACE is an important molecule in the renin-angiotensin system (RAS) (67) and also plays an important physiological role in regulating blood pressure (68). Beyazit et al (67) found that serum ACE levels were significantly higher in patients with ECC (56.6 \pm 27.4 units/1) compared with choledocolithiasis patients $(32.9 \pm 14.6$ units/l) and control groups $(28.6 \pm 10.6$ units/1). Circulating ACE in the RAS may be associated with ECC development through the formation of a local environment that is enriched with cytokines and other growth factors, possibly promoting cholangiocyte turnover.

Vitamin D3. The effects of vitamin D3 have been investigated in various tumors (69) and vitamin (1,25(OH)2D) exhibits anti-proliferative effects in several cancers (70). Kennedy et al (70) found the expression of the vitaminD3 receptor and cytochrome $\mathrm{P} 450$, family 24 , subfamily A, polypeptide 1 , an enzyme that degrades vitamin D3, increased in CCA lines and biopsy samples compared with controls. The expression of cytochrome P450, family 27, subfamily B, polypeptide 1 , an enzyme that synthesizes vitamin D3 and signals via the nuclear vitamin D3 receptor, was decreased or unchanged. In this study, it was suggested that modulation of vitamin D3 synthesis may be important in the management of CCA. As a result, the possibility of a change in serum level of vitamin D3 has been considered, and monitoring the level is a probable method for managing CCA. A large number of studies is required to explore this hypothesis.

Estrogen. Estrogenic response is known to be a promoting factor in the progression of certain cancers. Hunsawong et al (71) identified a significant increase in the serum estrogen level of male CCA patients. The levels were positively correlated with serum bilirubin and alkaline phosphatase, and negatively correlated with albumin. Estrogen also demonstrated an association with the survival times of patients.

$\gamma$-Glutamyltransferase (GGT). GGT is one of the enzymes used for the evaluation of liver function. Yin et al (72) found that GGT level was an independent predictor of tumor recurrence in ICC patients. Elevation of serum GGT levels is an indicator of aggressive tumor behavior and a predictor of poor clinical outcomes.

$C$-reactive protein $(C R P)$ and albumin. CRP and albumin are commonly tested for in serum biochemical examinations. The Glasgow prognostic score (GPS), an inflammation-based prognostic score (73), is useful in predicting the outcome for a variety of cancers. In ECC patients, $>10 \mathrm{mg} / 1 \mathrm{CRP}$ and $<35 \mathrm{~g} / 1$ hypoalbuminemia are allocated a score of 2 , and one or none of these abnormalities is allocated a score of 1 or 0 , respectively. As a result, increased GPS was found to be an independent predictive factor with poor prognosis in ECC by a Cox model (74). 


\section{Summary}

Local invasiveness and regional lymph node and distant organ metastasis is accountable for CCA morbidity and mortality (75). CCA is often diagnosed at an advanced stage (76). As numerous useful examination methods are invasive and dangerous, safer methods are required. Currently, there are no satisfactory serum makers that can accurately differentiate CCA from other diseases. The present review discussed certain traditional and novel serum makers that could aid in the diagnosis of CCA and in determining the prognosis of CCA, but the sensitivity and specificity of these markers are far from the present expectations. In future studies, it is necessary for additional useful serum makers to be identified.

Currently, there are certain methods for investigating novel serum markers, including proteomics, lipidomics, glycomics and microRNA (mi-RNA). As carcinogenesis possesses a complex and long progression, it will exhibit a series of serum characteristics in the process. Searching for markers using animal models that mimic the development of the disease is, perhaps, an indispensable step and, during the progression of the disease, observing the changes in the serum is also essential.

\section{References}

1. Khan SA, Davidson BR, Goldin RD, et al: Guidelines for the diagnosis and treatment of cholangiocarcinoma: an update. Gut 61: 1657-1669, 2012.

2. Gentilini A, Rombouts K, Galastri S, et al: Role of the stromal-derived factor-1 (SDF-1)-CXCR4 axis in the interaction between hepatic stellate cells and cholangiocarcinoma. J Hepatol 57: 813-820, 2012.

3. Limpaiboon T: Epigenetic aberrations in cholangiocarcinoma: potential biomarkers and promising target for novel therapeutic strategies. Asian Pac J Cancer Prev 13: 41-45, 2012.

4. Boonjaraspinyo S, Wu Z, Boonmars T, Kaewkes S, et al: Overexpression of PDGFA and its receptor during carcinogenesis of Opisthorchis viverrini-associated cholangiocarcinoma. Parasitol Int 61: 145-150. 2012.

5. Buranrat B, Chau-in S, Prawan A, et al: NQO1 Expression Correlates with Cholangiocarcinoma Prognosis. Asian Pac J Cancer Prev 13: 131-136, 2012.

6. Taylor-Robinson SD, Toledano MB, Arora S, et al: Increase in mortalityrates for intrahepatic cholangiocarcinoma in England and Wales 1968-1998. Gut 48: 816-820, 2001.

7. Tanaka M, Tanaka H, Tsukuma H, et al: Risk factors for intrahepatic cholangiocarcinoma: a possible role of hepatitis B virus. Journal of Viral Hepatitis 17: 742-748, 2010.

8. Li M, Li J, Li P, et al: Hepatitis B virus infection increases the risk of cholangiocarcinoma: a meta-analysis and systematic review. J Gastroenterol Hepatol 27: 1561-1568, 2012.

9. Sempoux C, Jibara G, Ward SC, et al: Intrahepatic cholangiocarcinoma: new insights in pathology. Semin Liver Dis 31: 49-60, 2011

10. Suarez-Munoz MA, Fernandez-Aguilar JL, Sanchez-Perez B, et al: Risk factors and classifications of hilar cholangiocarcinoma. World J Gastrointest Oncol 15: 132-138, 2013.

11. Zhou Y, Zhou Q, Lin Q, et al: Evaluation of risk factors for extrahepatic cholangiocarcinoma: $\mathrm{ABO}$ blood group, hepatitis $\mathrm{B}$ virus and their synergism. Int J Cancer 15: 1867-1875, 2013.

12. Sawanyawisuth K: Genes and cholangiocarcinoma. Southeast Asian J Trop Med Public Health 40: 701-712, 2009.

13. Fava G: Molecular mechanisms of cholangiocarcinoma. World J Gastrointest Pathophysiol 15: 12-22, 2010.

14. Briggs CD, Neal CP, Mann CD, et al: Prognostic molecular markers in cholangiocarcinoma: A systematic review. Eur J Cancer 45: 33-47, 2009.

15. Borger DR, Tanabe KK, Fan KC, et al: Frequent mutation of isocitrate dehydrogenase (IDH) 1 and IDH 2 in cholangiocarcinoma identified through broad-based tumor genotyping. Oncologist 17: 72-79, 2012 .
16. Subimerb C, Pinlaor S, Lulitanond V, et al: Circulating CD14+CD16+ monocyte levels predict tissue invasive character of cholangiocarcinoma. Clin Exp Immunol 161: 471-479, 2010.

17. Gu FM, Gao Q, Shi GM, et al: Intratumoral IL-17+ cells and neutrophils show strong prognostic significance in intrahepatic cholangiocarcinoma. Ann Surg Oncol 19: 2506-2514, 2012.

18. Utispan K, Thuwajit P, Abiko Y, et al: Gene expression profiling of cholangiocarcinoma-derived fibroblast reveals alterations related to tumor progression and indicates periostin as a poor prognostic marker. Mol Cancer 9: 1-20, 2010.

19. Keeratichamroen S, Leelawat K, Thongtawee T, et al: Expression of CD24 in cholangiocarcinoma cells is associated with disease progression and reduced patient survival. Int J Oncol 39: 873-881, 2011.

20. Yonglitthipagon $\mathrm{P}$, Pairojkul C, Chamgramol $\mathrm{Y}$, et al: Up-regulation of annexin A2 in cholangiocarcinoma caused by Opisthorchis viverrini and its implication as a prognostic marker. Int J Parasitol 40: 1203-1212, 2010.

21. Khan SA, Davidson BR, Goldin R, et al: Guidelines for the diagnosis and treatment of cholangiocarcinoma: consensus document. Gut 51: 1-9, 2002.

22. Li YG and Zhang N: Clinical significance of serum tumour M2-PK and CA19-9 detection in the diagnosis of cholangiocarcinoma. Dig Liver Dis 41: 605-608, 2009.

23. Leelawat K, Narong S, Wannaprasert J, et al: Prospective study of MMP7 serum levels in the diagnosis of cholangiocarcinoma. World J Gastroenterol 16: 4697-4703, 2010.

24. Singh S, Tang SJ, Sreenarasimhaiah J, et al: The clinical utility and limitations of serum carbohydrate antigen (CA19-9) as a diagnostic tool for pancreatic cancer and cholangiocarcinoma. Dig Dis Sci 56: 2491-2496, 2011.

25. Jo JH, Chung MJ, Park JY, et al: High serum CA19-9 levels are associated with an increased risk of cholangiocarcinoma in patients with intrahepatic duct stones: a case-control study. Surg Endosc 27: 4210-4216, 2013.

26. Wannhoff A, Hov JR, Folseraas T, et al: FUT2 and FUT3 genotype determines CA19-9 cut-off values for detection of cholangiocarcinoma in patients with primary sclerosing cholangitis 59: 1278-1284, 2013.

27. Sinakos E, Saenger AK, Keach J, et al: Many patients with primary sclerosing cholangitis and increased serum levels of carbohydrate antigen 19-9 do not have cholangiocarcinoma. Clin Gastroenterol Hepatol 9: 434-439, 2011.

28. Liu SL, Song ZF, Hu QG, et al: Serum carbohydrate antigen (CA) 19-9 as a prognostic factor in cholangiocarcinoma: a meta-analysis. Front Med China 4: 457-462, 2010.

29. Juntermanns B, Radunz S, Heuer M, et al: Tumor markers as a diagnostic key for hilar cholangiocarcinoma. Eur J Med Res 15: 357-361, 2010.

30. Silsirivanit A, Araki N, Wongkham C, et al: A novel serum carbohydrate marker on mucin 5AC: values for diagnostic and prognostic indicators for cholangiocarcinoma. Cancer 117: 3393-3403, 2011.

31. Sawanyawisuth K, Silsirivanit A, Kunlabut K, et al: A novel carbohydrate antigen expression during development of Opisthorchis viverrini-associated cholangiocarcinoma in golden hamster: a potential marker for early diagnosis. Parasitol Int 61: 151-154, 2012.

32. Silsirivanit A, Araki N, Wongkham C, et al: CA-S27: A novel Lewis a associated carbohydrate epitope is diagnostic and prognostic for cholangiocarcinoma. Cancer Sci 104: 1278-1284, 2013

33. Tao LY, Cai L, He XD, et al: Comparison of serum tumor markers for intrahepatic cholangiocarcinoma and hepatocellular carcinoma. Am Surg 76: 1210-1213, 2010.

34. Leelawat K, Sakchinabut S, Narong S, et al: Detection of serum MMP-7 and MMP-9 in cholangiocarcinoma patients: evaluation of diagnostic accuracy. BMC Gastroenterol 9: 1-8, 2009.

35. Prakobwong S, Charoensuk L, Hiraku Y, et al: Plasma hydroxyproline, MMP-7 and collagen I as novel predictive risk markers of hepatobiliary disease-associated cholangiocarcinoma. Int J Cancer 131: E416-E424, 2012.

36. Brewer HB Jr, Fairwell T, LaRue A, et al: The amino acid sequence of human APOA-I, an apolipoprotein isolated from high density lipoproteins. Biochem Biophys Res Commun 80: 623-630, 1978

37. Wang X, Dai S, Zhang Z, et al: Characterization of apolipoprotein A-I as a potential biomarker for cholangiocarcinoma. Eur J Cancer Care (Engl) 18: 625-635, 2009.

38. Leelawat K, Narong S, Wannaprasert J, et al: Serum NGAL to clinically distinguish cholangiocarcinoma from benign biliary tract diseases. Int J Hepatol 2011: 1-6, 2011. 
39. Flower DR: The lipocalin protein family: structure and function Biochem J 318: 1-14, 1996.

40. Srisomsap C, Sawangareetrakul P, Subhasitanont P, et al: Proteomic Studies of Cholangiocarcinoma and Hepatocellular Carcinoma Cell Secretomes. J Biomed Biotechnol 2010: 1-18, 2010.

41. Jie Z, Cai Y, Yang W, et al: Protective effects of $\alpha 1$-antitrypsin on acute lung injury inrabbits induced by endotoxin. Chin Med J (Engl) 116: 1678-1682, 2003.

42. Jamnongkan $\mathrm{W}$, Techasen $\mathrm{A}$, Thanan $\mathrm{R}$, et al: Oxidized alpha-1 antitrypsin as a predictive risk marker of opisthorchiasis-associated cholangiocarcinoma. Tumour Biol 34: 695-704, 2013.

43. Sriwanitchrak P, Viyanant V, Chaijaroenkul W, et al: Proteomics analysis and evaluation of biomarkers for detection of cholangiocarcinoma. Asian Pac J Cancer Prev 12: 1503-1510, 2011.

44. Tang W, Inagaki Y, Kokudo N, et al: KL-6 mucin expression in carcinoma of the ampulla of Vater: Association with cancer progression. World J Gastroenterol 11: 5450-5454, 2005.

45. $\mathrm{Xu} \mathrm{H}$, Inagaki $\mathrm{Y}$, Tang $\mathrm{W}$, et al: Elevation of serum KL-6 mucin levels in patients with cholangiocarcinoma. Hepatogastroenterology 55: 2000-2004, 2008.

46. Khoontawad J, Hongsrichan N, Chamgramol Y, et al: Increase of exostosin 1 in plasma as a potential biomarker for opisthorchiasis-associated cholangiocarcinoma. Tumour Biol 35 1029-1039, 2013.

47. Tian M, Cui YZ, Song GH, et al: Proteomic analysis identifies MMP-9, DJ-1 and A1BG as overexpressed proteins in pancreatic juice from pancreatic ductal adenocarcinoma patients. BMC Cancer 8: 1-11, 2008.

48. Jackson D, Craven RA, Hutson RC, et al: Proteomic profiling identifies afamin as a potential biomarker for ovarian cancer. Clin Cancer Res 13: 7370-7379, 2007.

49. Dieplinger H, Ankerst DP, Burges A, et al: Afamin and Apolipoprotein A-IV: Novel protein markers for ovarian cancer. Cancer Epidemiol Biomarkers Prev 18: 1127-1133, 2009.

50. Dieplinger B, Egger M, Gabriel C, et al: Analytical characterization and clinical evaluation of an enzyme-linked immunosorbent assay for measurement of afamin in human plasma. Clin Chim Acta 425: 236-241, 2013.

51. Tolek A, Wongkham C, Proungvitaya S, et al: Serum $\alpha 1 \beta$-glycoprotein and afamin ratio as potential diagnostic and prognostic markers in cholangiocarcinoma. Exp Biol Med (Maywood) 237: 1142-1149, 2012.

52. Zhang X, Xiao Z, Liu X, et al: The potential role of ORM2 in the development of colorectal cancer. PLoS One 7: 1-7, 2012.

53. Nagahara M, Nishida N, Iwatsuki M, et al: Kinesin 18A expression: clinical relevance to colorectal cancer progression. Int J Cancer 129: 2543-2552, 2011.

54. Rucksaken R, Khoontawad J, Roytrakul S, et al: Proteomic analysis to identify plasma orosomucoid 2 and kinesin 18A as potential biomarkers of cholangiocarcinoma. Cancer Biomark 12: 81-95, 2012.

55. Wang Y, Zhou B, Li J, et al: Inhibitors of 5-lipoxygenase inhibit expression of intercellular adhesion molecule-1 in human melanoma cells. Acta Pharmacol Sin 25: 672-677, 2004.

56. Touvier M, Fezeu L, Ahluwalia N, et al: Pre-diagnostic levels of adiponectin and soluble vascular cell adhesion molecule-1 are associated with colorectal cancer risk. World J Gastroenterol 18 : 2805-2812, 2012

57. Song WB, Lv YH, Zhang ZS, et al: Soluble intercellular adhesion molecule-1, D-lactate and diamine oxidase in patients with infl ammatory bowel disease. World J Gastroenterol 15: 3916-3919, 2009

58. Janan M, Proungvitaya S, Limpaiboon T, et al: Serum adhesion molecule-1 (icam-1) as a potential prognostic marker for cholangiocarcinoma patients. Asian Pac J Cancer Prev 13: 107-114, 2012.
59. Schneider J, Bitterlich N and Schulze G: Improved sensitivity in the diagnosis of gastro-intestinal tumors by Fuzzy Logic-based tumor marker profiles including the tumor M2-PK. Anticancer Res 25: 1507-1515, 2005.

60. Yin L, Wang X, Luo C, et al: The value of expression of M2-PK and VEGF in patients with advanced gastric cancer. Cell Biochem Biophys 67: 1033-1039, 2013.

61. Koss K, Harrison RF, Gregory J, et al: The metabolic marker tumour pyruvate kinase type M2 (tumour M2-PK) shows increased expression along the metaplasia-dysplasia-adenocarcinoma sequence in Barrett's oesophagus. J Clin Pathol 57: $1156-1159,2004$

62. Wu G, Bazer FW, Burghardt RC, et al: Proline and hydroxyproline metabolism: implications for animal and human nutrition. Amino Acids 40: 1053-1063, 2011

63. Jagtap VR and Ganu JV: Effect of antiresorptive therapy on urinary hydroxyproline in postmenopausal osteoporosis. Indian J Clin Biochem 27: 90-93, 2012.

64. Kristiansen TZ, Harsha HC, Grønborg M, et al: Differential membrane proteomics using 180-labeling to identify biomarkers for cholangiocarcinoma. J Proteome Res 7: 4670-4677, 2008.

65. Li W, Wang X, Li B, et al: Diagnostic significance of overexpression of Golgi membrane protein 1 in prostate cancer. Urology 80: e1-e7, 2012.

66. Chen MH, Jan YH, Chang PM, et al: Expression of GOLM1 correlates with prognosis in human hepatocellular carcinoma. Ann Surg Oncol 20: 616-624, 2013.

67. Beyazit Y, Purnak T, Suvak B, et al: Increased ACE in extrahepatic cholangiocarcinoma as a clue for activated RAS in biliary neoplasms. Clin Res Hepatol Gastroenterol 35: 644-649. 2011.

68. Liu JB, Yu ZP, Zhao WZ, et al: Liquid chromatographic assay of peptides activity with inhibiting angiotensin converting enzyme. Chem Res Chin Univ 26: 712-716, 2010.

69. Kósa JP, Horváth P, Wölfling J, et al: CYP24A1 inhibition facilitates the anti-tumor effect of vitamin D3 on colorectal cancer cells. World J Gastroenterol 19: 2621-2628, 2013.

70. Kennedy L, Baker K, Hodges K, et al: Dysregulation of vitamin D3 synthesis leads to enhanced cholangiocarcinoma growth. Dig Liver Dis 45: 316-322, 2013

71. Hunsawong T, Singsuksawat E, In-chon N, et al: Estrogen is increased in male cholangiocarcinoma patients' serum and stimulates invasion in cholangiocarcinoma cell lines in vitro. J Cancer Res Clin Oncol 138: 1311-1320, 2012.

72. Yin X, Zheng SS, Zhang BH, et al: Elevation of serum c-glutamyltransferase as a predictorn of aggressive tumor behaviors and unfavorable prognosis in patients with intrahepatic cholangiocarcinoma: analysis of a large monocenter study. Eur J Gastroenterol Hepatol 25: 1408-1414, 2013

73. Forrest LM, McMillan DC, McArdle CS, et al: Evaluation of cumulative prognostic scores based on the systemic inflammatory response in patients with inoperable non-small-cell lung cancer. Br J Cancer 89: 1028-1030, 2003.

74. Oshiro Y, Sasaki R, Fukunaga K, et al: Inflammation-based prognostic score is a useful predictor of postoperative outcome in patients with extrahepatic cholangiocarcinoma. J Hepatobiliary Pancreat Sci 20: 389-395, 2013.

75. Specht S, Isse K, Nozaki I, et al: SPRR2A expression in cholangiocarcinoma increases local tumor invasiveness but prevents metastasis. Clin Exp Metastasis 30: 877-890, 2013.

76. Sato Y, Harada K, Sasaki M, et al: Heat shock proteins 27 and 70 are potential biliary markers for the detection of cholangiocarcinoma. Am J Pathol 180: 123-130, 2012. 